\title{
Trauma de tórax. Experiencia de 5 años
}

\section{Chest trauma. A five-year experience}

\author{
Efrén Flores-Álvarez ${ }^{1 *}$, Dorian Y. García-Ortega², Efrén Flores-Tavizón ${ }^{3}$, Ramiro Y. Flores-Tavizón ${ }^{3}$, \\ Nancy Aguilar-Hernández ${ }^{3}$ y José A. Rodríguez-Osuna ${ }^{2}$ \\ ${ }^{1}$ Departamento de Enseñanza e Investigación, Centenario Hospital Miguel Hidalgo, ISEA; ${ }^{2}$ Departamento de Cirugía, Centenario Hospital Miguel \\ Hidalgo, ISEA; ${ }^{3}$ Universidad Autónoma de Aguascalientes. Aguascalientes. México
}

\section{Resumen}

Antecedentes: Las lesiones torácicas representan la cuarta parte de las lesiones traumáticas y causan el $50 \%$ de las muertes por traumatismos. Objetivo: Analizar la experiencia en traumatismo de tórax. Pacientes y métodos: Estudio retrospectivo que incluye a los pacientes con traumatismo torácico atendidos durante un periodo de cinco años. Resultados: Fueron 67 pacientes, con edad de $46.5 \pm 30$ años, $88.1 \%$ hombres y $11.9 \%$ mujeres. El traumatismo fue contuso en $59.7 \%$ y penetrante en $40.3 \%$. El mecanismo fue por objeto punzo-cortante en $35.8 \%$, lesiones por accidente automovilístico en $34.3 \%$ y lesiones por arma de fuego en 4.5\%. Las lesiones torácicas fueron neumotórax (53.7\%), hemoneumotórax (28.4\%), hemotórax (9\%), contusión pulmonar (3\%) y tórax inestable (1.5\%). El $80.6 \%$ de los casos fueron tratados con una sonda pleural, el $6 \%$ toracotomía y el $13.4 \%$ manejo expectante. El $82.1 \%$ cursó sin complicaciones. La mortalidad fue del $7.5 \%$. Conclusiones: Las principales causas de traumatismo torácico son las lesiones por arma blanca y los accidentes vehiculares. La mayoría se resuelven con la colocación de drenajes pleurales.

Palabras clave: Traumatismo de tórax. Sonda pleural. Toracotomía. Neumotórax. Hemoneumotórax.

\section{Abstract}

Background: Chest injuries accounts for a quarter of traumatic injuries and cause $50 \%$ of trauma-related deaths. Objective: To assess the experience in chest trauma at our hospital. Patients and methods: Retrospective study which included all chest trauma patients admitted in a five year period. Results: A total of 67 patients, $88.1 \%$ male and $11.9 \%$ female, with mean age of $46.5 \pm 30$ years. Thoracic trauma was blunt at $59.7 \%$ and penetrating at $40.3 \%$. The mechanism of trauma were stab wound $35.8 \%$, blunt trauma by motor vehicle accident 34.3 and gunshots $4.5 \%$. Chest injuries were pneumothorax $53.7 \%$, hemoneueumothorax $28.4 \%$, hemothorax $9 \%$, lung contusion $3 \%$ and flail chest $1.5 \%$. Treatment consisted in $80.6 \%$ a pleural tube, thoracotomy $6 \%$ and $13.4 \%$ expectant management. The average time of pleural drainage was 4.5 days. Mortality was $7.5 \%$. Conclusions: The main causes of chest trauma were stab wounds and blunt trauma by motor vehicle accidents. Most patients were treated by means of chest tube drainage.

Key words: Chest trauma. Pleural tube. Thoracotomy. Pneumothorax. Hemoneumothorax.

Correspondencia:

*Efrén Flores-Álvarez

E-mail: efflorez@ $@$ hotmail.com
Fecha de recepción: 17-02-2020

Fecha de aceptación: 11-03-2021

DOI: 10.24875/RHJM.20000030
Disponible en internet: 22-06-2021 Rev Hosp Jua Mex. 2021;88(2):45-49

www.revistahospitaljuarez.com 1405-9622/@ 2021 Sociedad Médico-Quirúrgica del Hospital Juárez de México, A.C. Publicado por Permanyer. Este es un artículo open access bajo la licencia CC BY-NC-ND (http://creativecommons.org/licenses/by-nc-nd/4.0/). 


\section{Introducción}

El traumatismo continúa siendo una de las principales causas de muerte en la población en general. En series estadounidenses se reportan hasta 140,000 muertes anuales por causa del traumatismo. La gran mayoría de los casos de traumatismo torácico se logra controlar con maniobras simples como una pleurostomía y de un 10 a un $15 \%$ de los pacientes llegan a requerir una resolución quirúrgica con toracotomía'.

Las lesiones del tórax corresponden a una cuarta parte de las lesiones traumáticas en general y el 50\% de las muertes por traumatismo. Las lesiones torácicas se clasifican en: traumatismo cerrado, penetrante o ambos; que generalmente ocurren como resultado de accidentes automovilísticos, lesiones por proyectil de arma de fuego o instrumento punzo-cortante, caídas de altura o compresión torácica por aplastamiento. Las lesiones torácicas ocasionadas por un traumatismo penetrante representan el $75 \%$ de las muertes relacionadas con traumatismo torácico ${ }^{2-4}$. Las estructuras lesionadas pueden incluir la pared torácica, los pulmones, el corazón, los grandes vasos y el diafragma. El neumotórax a tensión, el hemotórax masivo y el taponamiento cardiaco son lesiones potencialmente mortales que pueden ocurrir como resultado de un traumatismo de tórax. El tratamiento recomendado para estos pacientes se hace bajo los lineamientos establecidos por el ATLS (Advanced Trauma Life Support) $)^{5}$.

El diagnóstico y tratamiento de los traumatismos torácicos dependen en su mayor parte de la magnitud de la lesión y de la evidencia de lesiones asociadas. En la mayoría de los pacientes con lesiones por traumatismo de tórax resulta factible su resolución en forma conservadora, son manejados con buenos resultados mediante el drenaje de cavidad pleural con sondas o tubos pleurales ${ }^{3,6,7}$.

En el traumatismo se presentan tres picos de mortalidad: uno inicial, que se da en los primeros minutos al traumatismo (representa un $50 \%$ de la mortalidad global), un segundo pico ocurre en las tres horas siguientes al traumatismo (ocurren un $20 \%$ de las muertes) y un tercer pico corresponde a la mortalidad tardía (representa el $30 \%$ de la mortalidad). Se ha considerado a la primera hora luego del traumatismo como el "periodo de oro", durante el cual con un manejo adecuado se puede reducir la mortalidad del segundo y tercer periodos s,9. $^{8,}$.

Las caídas y los accidentes vehiculares son las causas más comunes de traumatismo torácico en la literatura internacional ${ }^{6-8,10}$. En nuestro medio aparecen como una de las causas mas frecuentes, aunadas a las lesiones producidas por arma blanca, producto de riñas y asaltos.

El objetivo del trabajo es analizar la experiencia en el manejo traumatismo de tórax en el departamento de cirugía de nuestro hospital en un periodo de cinco años.

\section{Material y método}

Se realizó un estudio retrospectivo, observacional, transversal, descriptivo y analítico. Fueron incluidos todos los pacientes mayores de 16 años con diagnóstico de traumatismo torácico que ingresaron al Centenario Hospital Miguel Hidalgo del ISEA en el periodo comprendido del 1 de enero de 2009 al 31 de diciembre de 2013. Las variables estudiadas fueron: edad, sexo, características de las lesiones torácicas, hallazgos radiográficos, el tiempo transcurrido entre la lesión y el ingreso al hospital, el mecanismo del traumatismo, el tipo de lesiones torácicas, el grado de severidad de las lesiones, la presencia de lesiones extratorácicas, el tratamiento efectuado en urgencias, el tratamiento en la unidad de terapia intensiva, el tipo de tratamiento quirúrgico, el tiempo de permanencia del drenaje pleural, el desarrollo de complicaciones, los días de estancia hospitalaria y la mortalidad.

La gravedad del traumatismo torácico fue evaluada mediante la escala de severidad de la lesión, que clasifica a los pacientes en traumatismo menor, moderado, serio, severo, crítico y máximo, y ha demostrado tener una buena correlación con la morbilidad, mortalidad y días de estancia hospitalaria ${ }^{11-13}$.

Se efectuó un análisis descriptivo de cada una de las variables en el paquete estadístico SPSS versión 17.0. Para las variables cualitativas nominales $u$ ordinales se calculó la frecuencia absoluta y porcentajes. Para las variables cuantitativas se estimaron medidas de tendencia central y de dispersión según el tipo de distribución de los datos.

\section{Resultados}

Fueron incluidos un total de 67 pacientes, el $88.1 \%$ $(n=59)$ fueron hombres y el $11.9 \%(n=8)$ mujeres. La media de la edad fue de $46.5 \pm 30$ años. El tipo de traumatismo fue contuso en 40 pacientes $(59.7 \%$ ) y penetrante en $27(40.3 \%)$. El tiempo transcurrido entre el momento en que ocurrió la lesión y el de la atención médica osciló entre 1 y 144 horas; la mayoría acudió 
al hospital durante las primeras 3 horas (primera hora, $29.9 \%$; segunda hora, $26.9 \%$, y tercera hora, $19.4 \%$ ). El mecanismo del traumatismo involucrado con mayor frecuencia fue por objeto punzo-cortante en 24 pacientes $(35.8 \%)$, accidente automovilístico en 23 pacientes (34.3\%) y por arma de fuego en 3 casos (4.5\%). Las causas según el tipo de traumatismo se muestran en la tabla 1.

Las lesiones torácicas más frecuentes fueron: neumotórax, 53.7\% ( $\mathrm{n}=36$ ); hemoneumotórax, $28.4 \%$ $(n=19)$; hemotórax, $9 \%(n=6)$; contusión pulmonar, $3 \%(n=2)$, y tórax inestable, $1.5 \%(n=1)$ (Tabla 2). El $34.3 \%(n=23)$ de los pacientes presentaron fracturas costales, el $4.5 \%(n=3)$ contusión pulmonar y el $4.5 \%$ $(n=3)$ presentaron más de dos lesiones. El $55.2 \%$ $(n=37)$ de los pacientes no presentaron otras lesiones torácicas 0 extratorácicas asociadas.

Las lesiones extratorácicas asociadas fueron: fracturas óseas en 10 pacientes (14.9\%), traumatismo craneoencefálico en 6 pacientes $(9 \%)$ y lesión a órganos abdominales en 3 pacientes (4.5\%) (Tabla 3). El 80.6\% de los pacientes $(n=54)$ fueron tratados con sonda pleural y sistema de sello de agua, se realizó toracotomía solo en el $6 \%$ de ellos $(n=4)$ y se estableció un manejo conservador no invasivo en el $13.4 \%(n=9)$. En el $70.2 \%(n=40)$ de los casos el drenaje pleural fue mantenido por menos de 5 días, el $28.1 \%$ entre 5 y 10 días $(n=16)$ y en el $1.7 \%(n=1)$ por más de 10 días.

Doce pacientes (17.9\%) recibieron manejo inicial en la unidad de cuidados intensivos, el resto fueron tratados en piso con medidas generales. Únicamente cuatro pacientes $(6 \%)$ desarrollaron complicaciones médicas a nivel torácico: síndrome de dificultad respiratoria del adulto en dos, neumonía en uno y atelectasia en un paciente. Nueve (13.4\%) de los pacientes ameritaron uso de aminas vasoactivas y 11 (16.4\%) requirieron apoyo mecánico ventilatorio. La mortalidad fue del $7.5 \%(n=5)$; una defunción fue relacionada directamente con el traumatismo torácico y las otras cuatro a lesiones en otros órganos y/o complicaciones de enfermedades asociadas propias del paciente.

La gravedad del traumatismo torácico la evaluamos con la escala de severidad de la lesión (Injury Severity Scale). Fueron considerados como traumatismo moderado el $55.2 \%$ de los casos, traumatismo grave que no pone en riesgo la vida el $17.9 \%$, traumatismo grave que pone en riesgo la vida el $17.9 \%$ y traumatismo crítico con supervivencia incierta el $9 \%$.
Tabla 1. Causas del traumatismo torácico

\begin{tabular}{|l|c|c|}
\hline Tipo & $\mathbf{n}$ & $\%$ \\
\hline Penetrante por objeto punzo-cortante & 24 & 35.8 \\
\hline Contuso automovilístico & 23 & 34.3 \\
\hline Contuso laboral & 6 & 9 \\
\hline Contuso por violencia física & 5 & 7.5 \\
\hline Otras contusiones & 6 & 9 \\
\hline Penetrante por proyectil de arma de fuego & 3 & 4.5 \\
\hline Total & 67 & 100 \\
\hline
\end{tabular}

Tabla 2. Lesiones torácicas más frecuentes

\begin{tabular}{|l|c|c|}
\hline Tipo & $\mathbf{n}$ & $\%$ \\
\hline Neumotórax & 36 & 53.7 \\
\hline Hemoneumotórax & 19 & 28.4 \\
\hline Hemotórax & 6 & 9 \\
\hline Contusión pulmonar & 2 & 3 \\
\hline Tórax inestable & 1 & 1.5 \\
\hline
\end{tabular}

Tabla 3. Lesiones torácicas y extratorácicas asociadas

\begin{tabular}{|l|c|c|}
\hline Tipo & $\mathbf{n}$ & $\%$ \\
\hline Lesiones óseas & 10 & 14.9 \\
\hline Lesiones óseas+TCE & 6 & 9 \\
\hline Lesiones de órganos abdominales & 3 & 4.5 \\
\hline TCE & 1 & 4.5 \\
\hline Lesiones óseas+abdominales & 1 & 4.5 \\
\hline Lesiones torácicas+lesiones óseas & 1 & 4.5 \\
\hline Lesiones torácicas+abdominales & 1 & 4.5 \\
\hline Lesiones torácicas+abdominales + óseas & 1 & 4.5 \\
\hline
\end{tabular}

TCE: traumatismo craneoencefálico.

\section{Discusión}

El traumatismo se considera un problema de salud pública en el mundo, en algunos lugares con un elevado índice de complicaciones y secuelas importantes. El traumatismo de tórax es una de las mayores causas de hospitalización en el mundo y corresponde al $10-15 \%$ de los traumas, con una mortalidad secundaria del $20-25 \%{ }^{14}$. La mayoría de los pacientes implicados 
en los accidentes que desencadenan un traumatismo torácico son de sexo masculino, debido a que los hombres se encuentran más expuestos a mecanismos violentos como riñas, agresiones físicas y accidentes automovilísticos. Las lesiones traumáticas continúan siendo una causa frecuente de mortalidad e incapacidad en personas en edad productiva, lo cual constituye un costo importante en horas laborales, así como pérdidas y gastos en atención médica ${ }^{1,15}$. La mayoría de nuestros pacientes fueron de sexo masculino, con una edad entre los 40 y 50 años, y afectación importante de su actividad laboral por un periodo prolongado.

El tipo de traumatismo de tórax es variable dependiendo del lugar y nivel de atención de las diversas series reportadas. El traumatismo penetrante es reportado como el más frecuente por algunos autores, llegando a ser hasta del $40 \%$ del total de los casos, otros estudios refieren tasas menores, entre el 20 y $30 \%$, y algunos otros, valores tan bajos como del $0.8 \%{ }^{16,17}$. El traumatismo penetrante se genera por la aplicación directa y abrupta de una fuerza mecánica en un área focal, los tres mecanismos principales son por proyectil de arma de fuego, por arma punzo-cortante y por empalamiento. Según el mecanismo traumático variará el grado de lesión provocada. En el caso de las heridas por proyectil de arma de fuego se deben considerar las características propias del proyectil, la biomecánica y sobre todo la velocidad con la que impacta a los tejidos debido a que habrá mayor intercambio de energía entre el tejido y el elemento lesivo. En el caso de lesiones por arma blanca, estas dependerán del sitio por el cual ingrese el arma y el trayecto y la profundidad alcanzada por el objeto implicado en el traumatismo. El empalamiento se presenta con mucha menor frecuencia y se produce por la penetración de una estaca u objeto elongado contundente que atraviesa el tórax ${ }^{18}$. Las lesiones por objetos punzo-cortantes son el mecanismo más frecuente por el que se producen las lesiones torácicas en los pacientes atendidos en nuestro hospital; las lesiones por proyectil de arma de fuego continúan siendo una causa menos frecuente, aunque ha ido aumentando en los últimos años.

El tratamiento basado en la inserción de una sonda pleural y la colocación de sello de agua es la alternativa más utilizada en el caso de las lesiones traumáticas del tórax, en diversas series se reporta en entre el 85 y $90 \%$ de los casos. En la presente serie, el $80.6 \%$ de los pacientes fueron tratados solo con sonda endopleural, tasa que coincide con lo publicado por Demirhan, et al. ${ }^{16}$, quienes reportan que el $73.6 \%$ de los pacientes con una patología traumática parecida a la de nuestra población requirieron únicamente dicho procedimiento. El tipo de lesión traumática ocasionada más frecuentemente en nuestros pacientes fue el neumotórax; en su mayoría fueron adecuadamente tratados con la colocación de tubos pleurales y sistemas de sello de agua, que fueron generalmente retirados en los primeros cinco días y egresados sin complicaciones.

La hemorragia intratorácica puede ser originada por lesiones de los vasos intercostales, mamarios o del parénquima pulmonar. La hemorragia secundaria a la lesión del parénquima pulmonar generalmente se autolimita debido a la baja presión en la circulación pulmonar, el alto contenido de tromboplastina del parénquima pulmonar y el efecto compresivo del pulmón colapsado sobre el sitio sangrante. En ocasiones se requiere una toracotomía de urgencia para tratar el hemotórax ${ }^{14,18,19}$, en nuestra serie solo se realizó en el $6 \%$ de los pacientes.

El traumatismo torácico se puede acompañar de lesiones en múltiples sistemas, lo cual condiciona una alta mortalidad en este grupo de pacientes 20,21 . En la presente serie encontramos que el $35.8 \%$ de los casos presentaban lesiones extratorácicas; la asociación más importante fue la asociación de traumatismo torácico con lesiones óseas y traumatismo craneoencefálico, presente en el $9 \%$ de los pacientes. En general, los pacientes con traumatismo de tórax sin lesiones en otros órganos evolucionan sin complicaciones durante su internamiento $y$, aunque su estancia hospitalaria no es en general breve, suelen egresarse antes del décimo día.

\section{Conclusión}

El traumatismo torácico representa una causa importante de ingreso al servicio de urgencias en nuestro hospital. El traumatismo secundario a lesiones por arma blanca y vehículos motorizados representan las principales causas de traumatismo torácico en pacientes atendidos en nuestra institución. La mayoría de los casos se resuelven con la colocación de drenajes pleurales, sistemas de sello de agua y cuidados de soporte.

\section{Agradecimientos}

A todos los integrantes del Departamento de Cirugía del Centenario Hospital Miguel Hidalgo, médicos adscritos al Servicio de Cirugía General y Cirugía Cardiotorácica, médicos residentes de Cirugía General e internos de pregrado, personal de enfermería y trabajo social. 


\section{Financiamiento}

Este estudio no ha recibido financiamiento externo.

\section{Conflicto de intereses}

Los autores declaran que no existe ningún conflicto de intereses.

\section{Responsabilidades éticas}

Protección de personas y animales. Los autores declaran que para esta investigación no se han realizado experimentos en seres humanos ni en animales.

Confidencialidad de los datos. Los autores declaran que han seguido los protocolos de su centro de trabajo sobre la publicación de datos de pacientes.

Derecho a la privacidad y consentimiento informado. Los autores han obtenido el consentimiento informado de los pacientes y/o sujetos referidos en el artículo.

\section{Bibliografía}

1. Meredith $\mathrm{H}$. Trauma thoracic. When and how to intervene. Surg Clin $N$ Am. 2007;87:95-118.

2. Valenzuela DM, Cancino AP, Cabezas SF, Donoso DG, de la Torre I. Experiencia en traumatismo torácico. Hospital Valparaíso. Rev Chilena Cir. 2003:55(5):4449-453.

3. Wells BJ, Roberts DJ, Grondin S, Navsaria PH, Kirkpatrick AW, Dunham MB, Ball ChG. To drain or not to drain? Predictors of tube thoracostomy insertion and outcomes associated with drainage of traumatic hemothoraces. Injury. 2015;46:1743-8.

4. Hernandez MC, Khatib ME, Prokop L, Zielinski MD, Aho JM. Complications in tube thoracostomy: Systematic review and meta-analysis. J Trauma Acute Care Surg. 2018;85(2):410-6.
5. American College of Surgeons Commitee on Trauma: Advanced Trauma Life Support.10 $0^{\text {th }}$ ed. American College of Surgeons; 2018.

6. Karmy-Jones R, Nathens A, Jurkovich GJ, Shatz DV, Brundage S, Wall MJ, et al. Urgent and emergent thoracotomy for penetrating chest trauma. J Trauma. 2004;56:664-69.

7. Bevis LC, Berg-Copas GM, Thomas BW, Vasquez DG, Wetta-Hall R, Brake D, et al. Outcomes of tube thoracostomies performed by advanced practice providers vs trauma surgeons. Am J Crit Care. 2008;17(4): 357-63.

8. Söderlund T, Ikonen A, Pyhältö T, Handolin L. Factors associated with in-hospital outcomes in 594 consecutive patients suffering from severe blunt chest trauma. Scand J Surg. 2014;104:115-20.

9. Cothren C, Moore EE. Emergency department thoracotomy for the critically injured patient: Objectives, indications, and outcomes. World $\mathrm{J}$ Emerg Surg. 2006;1:4

10. Martinus R, Krettek C, Otte D, Wiese SM, Stalp M, Ernst S, et al. Correlation between crash severity, injury severity, and clinical course in car occupants with thoracic trauma: A technical and medical study. J Trauma. 2001;50:10-6

11. Baker SP, O'Neill B, Haddon W Jr, Long WB. The Injury Severity Score: a method for describing patients with multiple injuries and evaluating emergency care. J Trauma. 1974;14:187-96.

12. Copes WS, Champion HR, Sacco WJ, Lawnick MM, Keast SL, Bain LW. The Injury Severity Score Revisited. J Trauma. 1988;28:69-77.

13. Narayanan R, Kumar S, Gupta A, Bansal VK, Sagar S, Singhal M, et al. An analysis of presentation, pattern and outcome of chest trauma patients at an Urban Level 1 Trauma Center. Indian J Surg. 2018;80(1):36-41.

14. Cuellar-Bahamón AM, Charry JD, Medina R, Chávarro O. Complicaciones del manejo quirúrgico de los pacientes con trauma penetrante de tórax en un hospital universitario al sur de Colombia. Panam J Trauma, Critical Care Emerg Surg. 2019:8(1):52-5.

15. Majercik S, Pieracci FM. Chest wall trauma. Thorac Surg Clin. 2017;27(2):113-21

16. Demirhan R, Onan B, Oz K, Halezeroglu S. Comprehensive analysis of 4205 patients with chest trauma: a 10-year experience. Interact Cardiovasc Thorac Surg. 2009;9(3):450-3.

17. Tariq UM, Faruque A, Ansari H, Ahmad M, Rashid U, Perveen S, et al. Changes in the patterns, presentation and management of penetrating chest trauma patients at a level II trauma centre in southern Pakistan over the last two decades. Interact Cardiovasc Thorac Surg. 2011;12(1):24-7.

18. Huh J, Wall MJ, Estrera AL, Soltero ER, Mattox KL. Surgical management of traumatic pulmonary injury. Am J Surg. 2003;186(6):620-4

19. Ludwig C, Koryllos A. Management of chest trauma. J Thorac Dis. 2017;9:S172-S177.

20. Hildebrand F, Giannoudis PV, Griensven MV, Zelle B, Ulmer B, Krettek $C$, et al. Management of polytraumatized patients with associated blunt chest trauma: a comparison of two European countries. Injury. 2005;36(2):293-302.

21. Chrysou K, Halat G, Hoksch B, Schmid RA, Kocher GJ. Lessons from a large trauma center: impact of blunt chest trauma in polytrauma patients-still a relevant problem? Scand J Trauma Resusc Emerg Med. 2017;25(1):42 